\title{
Creencias de los cuidadores de niños sobre la prevención de lesiones no intencionales, según sus experiencias
}

\section{Child caregivers' beliefs about preventing unintentional injuries, based on their experiences}

\author{
Rosa Elizabeth Sevilla-Godínez,* Javier Eduardo García-De Alba,* Blanca Miriam Torres-Mendoza* \\ * Departamento de Disciplinas Filosófico, Metodológicas e Instrumentales, Centro Universitario de Ciencias de la Salud, Universidad de \\ Guadalajara. Departamento de Neurociencias, Centro de Investigación Biomédica de Occidente, Instituto Mexicano del Seguro Social. México.
}

\begin{abstract}
RESUMEN
Introducción: Las costumbres, creencias y percepciones que el cuidador de niños ha adquirido en su vida influyen en su comportamiento preventivo. Objetivo: Identificar la representación social en cuidadores sobre la prevención de lesiones no intencionales $(\mathrm{LNI})$ en niños menores de seis años residentes en Guadalajara, México. Material y métodos: Estudio cualitativo en dos grupos focales, compuestos por cuidadores de niños en edad preescolar, quienes fueron seleccionados mediante muestreo teórico. Un grupo tenía experiencia anterior en LNI y el otro sin esta experiencia. Mediante un proceso de inducción analítica, a partir de las respuestas de los participantes se elaboró una matriz manual con las categorías y los códigos. Resultados: Los cuidadores con experiencia en LNI perciben de manera diferente el peligro y realizan acciones para prevenir lesiones. Los cuidadores sin experiencia requieren tener la certeza de que al prevenir no sucederá un LNI. Conclusiones: Las conductas de prevención están influenciadas por los significados sociales y culturales de los cuidadores, así como experiencias previas. La información que aquí se presenta puede ayudar a fortalecer programas dirigidos a prevenir LNI en niños.
\end{abstract}

Palabras clave: Prevención, lesiones no intencionales, cuidadores, creencias, estudio cualitativo.

\section{INTRODUCCIÓN}

Los cuidadores son una pieza fundamental en la seguridad de los niños, ${ }^{1}$ porque velan por su in-

\begin{abstract}
Introduction: Customs, beliefs and perceptions that caregivers have acquired in their lives influence their preventive behavior. Objective: To identify the social representation on the prevention of unintentional injuries (UI) among caregivers of children younger than six years old, in Guadalajara, Mexico. Material and methods: qualitative study in two focus groups composed of caregivers of preschoolers, who were selected by theoretical sampling. One group had previous experience in $\mathrm{UI}$ and the other without this experience. Through an analytical induction process, a matrix with the categories and codes was developed from the participants' responses. Results: Caregivers with experience in UI perceive danger differently and take actions to prevent injuries. Inexperienced caregivers need to be certain that by preventing an UI will not happen, in order to establish actions. Conclusions: Prevention behaviors are influenced by the social and cultural meanings of the caregivers, as well as previous experiences. The information presented here can help strengthen programs to prevent UI in children.
\end{abstract}

Keywords: Prevention, unintentional injuries, caregivers, beliefs, qualitative study.

tegridad física y tienen gran responsabilidad de mantener seguro el medio ambiente para prevenir lesiones no intencionales (LNI). ${ }^{2}$ Se ha señalado que los cuidadores carecen de reconocimiento de

Correspondencia: Rosa Elizabeth Sevilla-Godínez, rosbeth@gmail.com

Citar como: Sevilla-Godínez RE, García-De Alba JE, Torres-Mendoza BM. Creencias de los cuidadores de niños sobre la prevención de lesiones no intencionales, según sus experiencias. Rev Mex Pediatr. 2021; 88(2): 53-60. https://dx.doi.org/10.35366/101278 
la susceptibilidad de los niños bajo su cuidado en el hogar, lo cual puede favorecer la existencia de riesgos para lesiones. ${ }^{3}$

Las costumbres, creencias y percepciones que el cuidador ha adquirido en su vida influyen en su comportamiento y en las acciones que realiza en la prevención. ${ }^{4}$ Estas actitudes tienen de fondo una representación social que se construye y vive en consecuencia. ${ }^{5-7}$ Estas representaciones sociales se manifiestan en diferentes actitudes de inseguridad o riesgo. Por ejemplo, las lesiones de tránsito han sido estudiadas con la teoría de las representaciones sociales para dar cuenta de una serie de manifestaciones sociales que imperan entre los conductores. ${ }^{8}$ También las representaciones sociales han sido estudiadas en la crianza de los niños por ser una etapa en la cual se inculca y se educa al niño de acuerdo a las creencias y cultura de los padres de familia, por lo que, de manera implícita, se puede incluir el cuidado y seguridad del niño. ${ }^{9}$

La percepción del riesgo y las creencias que tengan los cuidadores pueden reducir la valoración del peligro y evitar la prevención. ${ }^{10}$ Por tanto, comprender cómo piensan los cuidadores sobre la prevención puede identificar las posibles limitantes para que no se lleven a cabo acciones de prevención, a pesar de contar con los recursos para hacerlo. Por tal motivo, el objetivo del estudio fue identificar la representación social de la prevención de LNI en niños que tienen sus cuidadores primarios, de acuerdo a experiencias previas de LNI.

\section{MATERIAL Y MÉTODOS}

Estudio cualitativo, fenomenológico aprobado por los Comités de Investigación, Ética en Investigación y Bioseguridad pertenecientes al Centro Universitario de Ciencias de la Salud de la Universidad de Guadalajara (Registro 18-76).

En este estudio, se buscaba conocer la representación social de los cuidadores de niños menores de seis años acerca de la prevención de LNI. Para tal efecto, como primer paso se realizó una entrevista semiestructurada con expertos en el área de pediatría (médicos y no médicos), de cuyas respuestas consensuadas se obtuvo el material para elaborar una guía de preguntas. Las preguntas eje fueron en torno a la estructura y dinámica de la representación social, así como al proceso de la prevención como cuidadores de niños.

La búsqueda de participantes se efectuó de la siguiente manera:
1. Se realizó una llamada al Consejo Estatal de Prevención de Accidentes en Jalisco (CEPAJ) para identificar la zona con mayor incidencia de LNI.

2. Se procedió a la búsqueda por Internet del listado oficial de jardines de niños de la zona metropolitana de Guadalajara, Jalisco.

3. Se identificaron cuatro jardines de niños que se encontraron dentro de la zona señalada por el CEPAJ.

4. Se seleccionaron al azar dos jardines, solicitando la autorización del trabajo de campo con la directora del plantel. Bajo este proceso, se descartaron los dos primeros. El tercero y cuarto fueron seleccionados para desarrollar el proyecto. Estos jardines de niños están ubicados en áreas geoeconómicas de estrato medio

En las dos escuelas se procedió a buscar a dos grupos focales que deberían cumplir con las siguientes características: 1) cuidadores de niños menores de seis años que no hubieran experimentado (hasta el momento de la investigación) una lesión intencional; y 2) cuidadores de niños menores de seis años que hubieran vivido una experiencia de LNI, en cualquier etapa de su vida como cuidadores. En estos grupos no se incluyeron niños.

El lugar para desarrollar el grupo focal fue el plantel de educación formal de los niños. La invitación a integrarse a los grupos focales la realizó la educadora de los grupos de preescolar. No hubo penalización o estímulo para tal efecto, por lo que de manera libre los cuidadores tomaron la decisión para participar en el estudio.

$\mathrm{Al}$ interior de cada grupo focal, se dialogó sobre el proyecto, se aceptó participar y firmar la carta de consentimiento informado y se autorizó que la sesión fuera grabada en audio.

Se utilizó una muestra teórica acumulada hasta llegar a la saturación de la información solicitada. En total se integraron 14 cuidadores (siete en cada grupo focal) conformados por papás, mamás o abuelas de los niños.

Cada grupo de estudio estuvo en sesiones separadas, con una sesión por cada grupo de 60 minutos en promedio.

Los criterios considerados de calidad y validez para la información de los grupos focales fueron:

1. El grupo terminó la guía de entrevista.

2. Todos los integrantes del grupo focal participaron.

3. El grupo otorgó consenso a los significados y sentidos resultantes. 
4. Cada tema que surgió fue cerrado y se generó consenso.

Las entrevistas de los grupos focales fueron conducidas por estudiante de doctorado y una licenciada en nutrición, quien apoyó en las cuestiones logísticas y en el registro en el cuaderno de notas sobre los gestos, movimientos o cualquier otro rasgo de los participantes de los grupos focales que pudieran enriquecer el diario de campo.

Para el análisis de la información, las grabaciones de audio de las sesiones de cada grupo focal fueron transcritas y analizadas para identificar las ideas centrales de cada párrafo. Se ubicaron los conceptos clave y se asignó una lista de otras ideas que se agruparon en esas grandes categorías. Se capturó la información para la identificación de una estructura temática. Después, mediante un proceso de inducción analítica, se determinó una matriz manual con categorías y en éstas se agruparon los códigos que las conformaron.

\section{RESULTADOS}

Se conformaron dos grupos focales de cuidadores de segundo grado de preescolar que diferían en la presencia o ausencia de una experiencia de LNI en niños. El grupo focal con LNI estuvo integrado por seis mamás y una abuela; el promedio de edad de los cuidadores fue de 42 años. En el grupo focal sin LNI participaron cinco mamás, un papá y una abuela con un promedio de edad de 35 años.
Se aplicó una inducción analítica a las entrevistas de los grupos focales.

De forma manual, se generaron los conceptos e ideas claves y se construyó la categoría de "significado de la prevención de LNI en los niños" y las diferencias y similitudes de los 23 códigos encontrados en esta categoría entre los dos grupos focales (Tabla 1).

Para ambos grupos, las LNI son imprevistas y forman parte de la vida. Además de que la imagen social que proyecta quien previene es de "alucinado" u "obsesivo".

Se encontró que los cuidadores tienen motivos a favor y en contra de la prevención de LNI, los cuales fueron agrupados de acuerdo al tipo de motivos en diferentes conceptos que se muestran en la Tabla 2.

\section{DISCUSIÓN}

En este estudio, los cuidadores de niños que han experimentado una LNI tienen mayor conciencia de las acciones que facilitan la prevención, por lo tanto, anclan el conocimiento de la experiencia vivida en sus conductas preventivas, contrario a lo señalado por otros autores. ${ }^{11}$ También los cuidadores que ya tuvieron la experiencia de una LNI presentan una forma distinta de ver la prevención y presentan argumentos opuestos a quienes no han vivido una LNI.

El grupo sin experiencia de LNI quiere tener la certeza absoluta de que si se previene no se lesionará el niño, lo cual impide tener una idea concreta de la prevención, y para ellos da lo mismo cuidar al niño o no.

Tabla 1: Diferencias y similitudes de los códigos de los grupos focales en relación con el concepto de prevención.

Significado de la prevención y de las LNI en niños

Similitudes

Diferencias
Códigos en cuidadores con niños que sí habian experimentado LNI

No intencionales
Es normal lesionarse
Evento inesperado
Estar alucinado
"Hasta que sucede se aprende"
Fragilidad
Cuestiones externas
Valentía
Ser controlador
Implica conflicto de pareja
Pensar implica modificar
Mejor no pienso

Es normal lesionars

Evento inesperado

Fragilidad

Valentía

Ser controlador

Pensar implica modificar

Mejor no pienso
Códigos en cuidadores con niños que no habian experimentado LNI

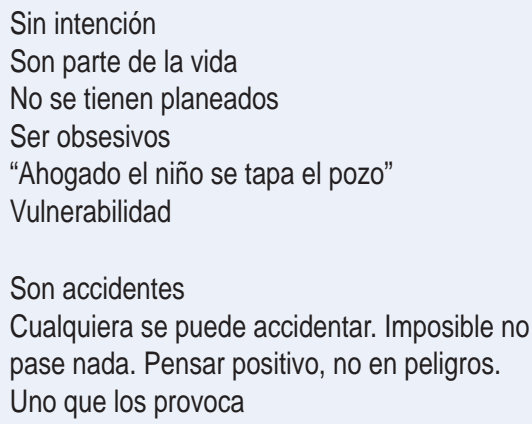

Sin intención

Son parte de la vida

No se tienen planeados

Ser obsesivos

"Ahogado el niño se tapa el pozo"

Vulnerabilidad

Son accidentes

Cualquiera se puede accidentar. Imposible no pase nada. Pensar positivo, no en peligros. Uno que los provoca

$\mathrm{LNI}=$ lesiones no intencionales. 
Tabla 2: Análisis de las diferencias de los dos grupos de acuerdo a los pros y contras para la prevención.

\begin{tabular}{|c|c|c|}
\hline \multirow[b]{2}{*}{ Conceptos } & \multicolumn{2}{|c|}{ Motivos para prevenir LNI } \\
\hline & A favor & En contra \\
\hline \multirow[t]{2}{*}{$\begin{array}{l}\text { Relacionados con la educación o } \\
\text { información al niño }\end{array}$} & Educar e informar al niño sobre LNI & $\begin{array}{l}\text { A pesar del cuidado, los niños se lesionan y, aun sin } \\
\text { cuidarlos no se lesionan }\end{array}$ \\
\hline & $\begin{array}{l}\text { Recomiendan comunicar los peligros que } \\
\text { existen en la casa }\end{array}$ & $\begin{array}{l}\text { Sobre o subestiman las capacidades de los niños } \\
\text { para superar las medidas de protección que } \\
\text { implementan }\end{array}$ \\
\hline \multirow[t]{4}{*}{$\begin{array}{l}\text { Relacionados con las prácticas y } \\
\text { habilidades de los cuidadores }\end{array}$} & $\begin{array}{l}\text { Se habla del cuidado que deben de tener } \\
\text { quienes cuidan a los niños }\end{array}$ & \\
\hline & $\begin{array}{l}\text { Señalan agudizar el sentido común o } \\
\text { instinto, para reconocer los peligros que de } \\
\text { otra manera pasarían desapercibidos }\end{array}$ & \\
\hline & $\begin{array}{l}\text { Recomiendan tener calma y detenerse un } \\
\text { tiempo para identificar peligros }\end{array}$ & \\
\hline & $\begin{array}{l}\text { Aceptan que la flojera y el cansancio } \\
\text { impiden que se activen en las conductas de } \\
\text { seguridad para los niños }\end{array}$ & \\
\hline Relacionadas al género del cuidador & $\begin{array}{l}\text { Identifican diferencias en las medidas de } \\
\text { prevención de acuerdo al género }\end{array}$ & No previenen, para evitar conflictos en la pareja \\
\hline \multirow[t]{2}{*}{$\begin{array}{l}\text { Relacionados con materiales y } \\
\text { productos peligrosos en el hogar }\end{array}$} & $\begin{array}{l}\text { El control de las cosas lo identifican como } \\
\text { una conducta favorable }\end{array}$ & \\
\hline & $\begin{array}{l}\text { Importante almacenar con seguridad } \\
\text { productos tóxicos o peligros que existen en } \\
\text { el hogar }\end{array}$ & \\
\hline Relacionados con el hogar & $\begin{array}{l}\text { Identifican que la estética del hogar puede } \\
\text { conllevar peligros ("hogar de adultos y no } \\
\text { hogar de niños") }\end{array}$ & \\
\hline \multirow[t]{2}{*}{ Relacionados con el modo de vida } & $\begin{array}{l}\text { Están conscientes que el estilo de vida, con } \\
\text { las rutinas y costumbres, no deja ver los } \\
\text { peligros }\end{array}$ & $\begin{array}{l}\text { Pensar en daño es encontrar qué modificar, lo cual } \\
\text { altera la comodidad }\end{array}$ \\
\hline & Relacionan la prevención con libertad & $\begin{array}{l}\text { Costo-beneficio: siempre inclinado al beneficio a } \\
\text { pesar del costo implícito }\end{array}$ \\
\hline Relacionados con actitudes & & No existe credibilidad en la prevención \\
\hline Generales & & $\begin{array}{l}\text { Se busca la certeza en que si se previene no se } \\
\text { lesionará } \\
\text { Hay una actitud de burla ante el peligro, al cual no se } \\
\text { le enfrenta como tal } \\
\text { La idea de prevención no se tiene presente en la } \\
\text { acciones del día a día (no hay planeación) } \\
\text { Los peligros se dejan hasta que suceden las cosas } \\
\text { Se percibe como "valiente" a quien ha sufrido una LNI }\end{array}$ \\
\hline
\end{tabular}

$\mathrm{LNI}=$ lesiones no intencionales.

De acuerdo con Giddens, ${ }^{12}$ cuando se previene se asume que existe vulnerabilidad y riesgo, de manera que la conciencia de la probabilidad de que se presente una LNI es lo que permite anticiparse para evitar esa eventualidad. ${ }^{13}$ Para los cuidadores sin experiencia de LNI, esta probabilidad no parece ser importante. Incluso adoptan una actitud estigmatizante ante quien previene ("obsesivo", "alucinado"), como se puede comprender en los siguientes comentarios: 
- Lety: “...obsesivo puede ser algunas personas que están así como hasta imaginando lo que puede pasar, o qué no puede pasar, entonces sobredimensionan alguna situación que para el resto de los adultos pudiera ser así como de ay, qué exageración”.

- Ara: "...mi mismo marido me dice, tú pareces ave de mal agüero, no hables de eso porque me estás trayendo la mala suerte,... te tachan de ave de mal agüero o de que estás alucinada”.

Asimismo, en ocasiones se burlan del peligro, lo que forma parte de los elementos encontrados en los discursos de los cuidadores que se interrelacionan que pueden llevar a una LNI (Figura 1).

En contraste, el grupo con experiencia de LNI, los argumentos para prevenir se sustentan en cuestiones de base sociocultural, de pérdida del confort personal:

- Ara: “...por comodidad tienes algún medicamento en tu cajón de tu buró... habría que revisar bien qué tienes... pues ahi yo le veo un peligro a esos cajoncitos que luego a los niños les da mucha curiosidad por abrir".

Además, sobre o subestiman al niño en sus capacidades:

- Lety: "Pues básicamente por negligencia o descuido... y a lo mejor... por menospreciar un poquito la capacidad del niño al decir, ah, no se le va a ocurrir o eso no le pasa".

Se estimaría que es necesaria la experiencia para que se pueda considerar a la prevención como una prioridad y tomar conciencia de ella. Justo así lo piensan los cuidadores.
- Adri: “...y desgraciadamente uno como humano aprende hasta que te pasan las cosas... porque muchas de las veces te dicen; oye, no hagas esto porque te puede pasar esto, ¿será cierto? Entonces, lo tienes quizás que pasar para creer".

Lo anterior conduce a la siguiente pregunta: ¿se tiene que sufrir para aprender? La respuesta es no. Se ha reportado que la experiencia de un grupo mejora los cuidados, ${ }^{13,14}$ en este caso particular, quienes ya han experimentado una LNI pueden transmitir el mensaje a quienes no han experimentado un evento y a partir de ahí tener certeza de que las LNI suceden.

Ante la frecuente defensa de que el riesgo es sólo probable, sería conveniente considerar el principio de responsabilidad de Hans Jonas. ${ }^{15}$ Si bien este principio está dirigido a la ciencia y tecnología, así como su efecto en la naturaleza y ecología, podría también aplicarse a la prevención. Jonas "invierte el significado del principio cartesiano de la duda", ${ }^{16} \mathrm{de}$ manera que, para efectos de la decisión, sugerimos manejar lo dudoso pero posible, como si fuera cierto. En este sentido, la probabilidad que es inherente al riesgo, y que los cuidadores argumentaron que es una de las razones por las cuales no previenen, pudiera ser anulada si se considera esta duda como cierta. Es decir, pensar no como riesgo probable, sino como peligro seguro, generaría acciones consecuentes porque estaría basada en una certeza del daño y no en una probabilidad del riesgo. Este es un argumento muy útil que puede manejarse ante quienes requieren una certeza para actuar:

- Paola: “... estoy segura que, si tienes una bolita de cristal y te dicen, hoy vas a chocar, pase lo que pase, vas y le compras la sillita que mucha gente nunca le
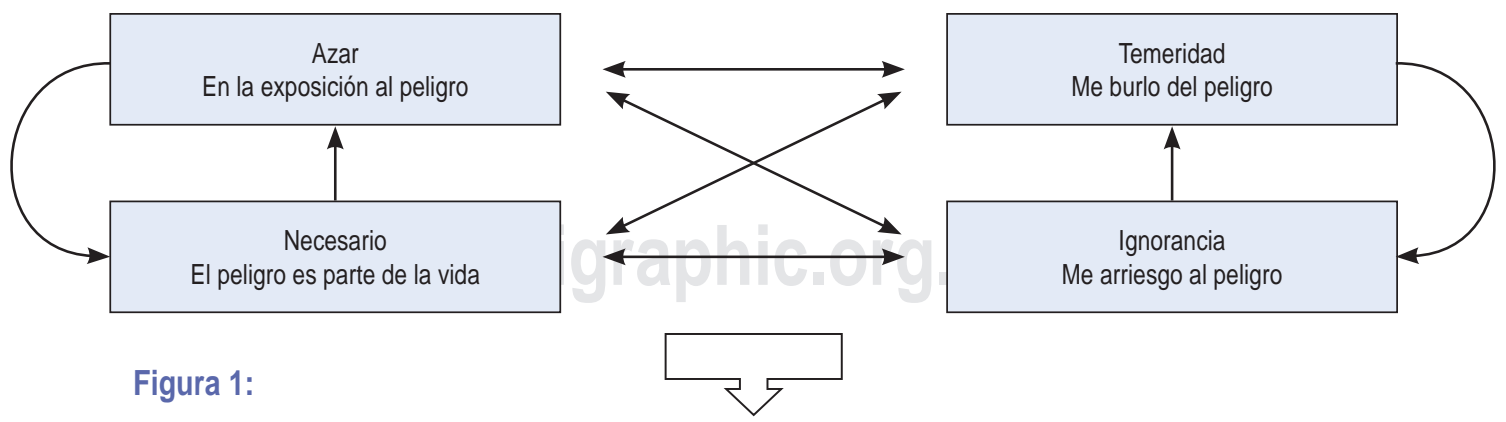

Figura 1:

Ocurrencia de lesiones no intencionales 
compró a su niño, aunque tenga ya cuatro años y se lo pondría, o no saldría".

Pareciera que sólo si el accidente es inminente entonces se previene, y como no se tiene la certeza de que suceda una LNI (lo cual es real), y no hay una garantía como tal de que no suceda una LNI (lo cual también es real), entonces, no se previene. Sin embargo, la ocurrencia de una LNI siempre está medida en una probabilidad, no en una certeza. El reto es lograr que se actúe ante la incertidumbre, pues en la vida cotidiana se transita siempre con incertidumbres, porque nada está garantizado de antemano. Una manera de hacer este cambio de la conducta humana es a través del consejo de quienes han experimentado una LNI en ninos, a fin de concientizar a quienes no lo han sufrido. ${ }^{12}$

Hans Jonas señala otro principio que también puede ser trasladado al ámbito de prevención de LNI, que es la "heurística del temor". ${ }^{15} \mathrm{Al}$ miedo se le interpreta como una emoción patológica que oscurece la conciencia y paraliza la voluntad. Es decir, el temor merma la capacidad reflexiva y volitiva del individuo, obstaculizando la buena deliberación, decisión y acción, algo que, en definitiva, impide el ejercicio de la responsabilidad. No obstante, el miedo o temor cumple una función heurística: descubre el bien a preservar a través del sentimiento (de miedo/temor) que produce el mal imaginado. Lamentablemente los cuidadores llegaron a decir que mejor no pensaban en el daño:

- Karla: “...esa falta de seguridad te hace sentirte ansioso y te hace sentirte como... en una situación en la que ya emocionalmente no puedes controlar las cosas y a veces es como mejor hacerte el ciego, el que no ves, el que no pasa nada porque al final creemos que ahora sí que ojos que no ven, corazón que no siente, es... más fácil sobrellevar eso".

Los cuidadores indicaron que, al pensar en el daño, sienten miedo, fragilidad y vulnerabilidad, los cuales son inhibidores porque implica modificar lo que pueda representar peligro en el hogar, que a su vez altera la comodidad, además de que esa modificación se hace para un evento futuro probable. Pensar así, favorece las condiciones para una LNI (Figura 2).

Bajo la perspectiva de la "heurística del temor", el miedo debiera ser el detonante que motive a actuar, al pensar en lo que no se quiere que pase. Es decir, los cuidadores no piensan lo que les hace sentir miedo. Sin embargo, es precisamente ese sentimiento de miedo o temor a lo que puede pasar (una LNI, la muerte), y que si se canaliza adecuadamente, debería provocar acciones para proteger la vida del niño. Ahora bien, si como algunos cuidadores señalan que las LNI son parte de la vida, entonces cabe hacer la pregunta ¿para qué prevenir? o, si se piensa que vivir con miedo no es vivir, habría que enfatizar que no se trata de vivir con miedo, sino que el miedo sea lo que active la prevención y una vez que se prevé, el miedo desaparece porque el peligro ya no está.

- Sara: "con cualquier cosita pasan los accidentes... son descuidos que, también yo digo que es parte de la vida".

\section{El instinto como recurso de prevención}

Los cuidadores consideran que el instinto les puede indicar que algo no está bien. El principio de precaución como "mandato de cautela" de Hans Jonas aplica en este sentido. ${ }^{16}$ Esta anticipación imaginante-emocional de un mal futuro tiene una virtualidad cognitiva, cuyo saber no es probatorio (es decir, no existe certeza de algo que todavía no ha ocurrido), pero del que sí se puede decir que es un saber-sentimiento premonitorio, anticipando que pueda ocurrir aquello que aún no ha ocurrido. Visto así, puede ayudar a no exponerse a riesgos para evitar la ocurrencia de una LNI para prevenir. ${ }^{17}$ Los cuidadores atribuyen que el instinto hace actuar, ante los peligros que de otra forma pudieran pasar desapercibidos:

- Karla: “...muchos papás... queremos rescatar esta parte del instinto y de regresar a lo básico como con los cuidados, nos perdemos entre tanto acelere y tanta cosa que no logramos esa estabilidad y no nos percatamos de todos los riesgos que puede haber en la casa”.

En las representaciones sociales, el sentido común ${ }^{18,19}$ forma parte de un conocimiento social que ha sido compartido, que incluye los valores y creencias que permiten comunicarse, actuar y racionalizar las acciones. ${ }^{20} \mathrm{El}$ sentido común aporta esa forma de actuar, con base en un conocimiento general adquirido y socialmente aprobado que puede anteponerse a la cultura de la evasión.

\section{Resiliencia en la prevención}

Pareciera que el aprendizaje que se ha obtenido de la experiencia de quienes han experimentado una LNI 


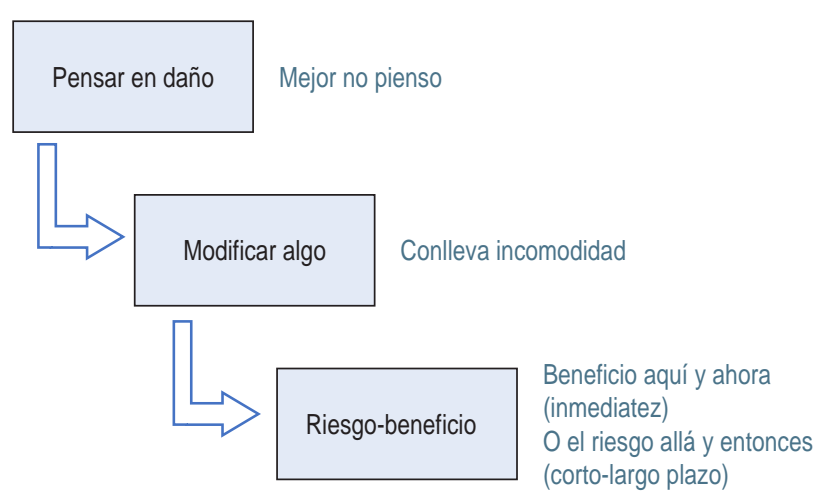

Fuente: Elaboración propia.

Figura 2: La forma de pensar el daño que origina las lesiones no intencionales, según los cuidadores.

se refleja en los cuatro aspectos potenciales para el desempeño resiliente: ${ }^{21,22}$

1) aprender de los hechos;

2) responder con el desempeño actual del aprendizaje de los hechos;

3) monitorear lo aprendido que genera daño; y

4) anticipar lo que puede pasar por el potencial riesgo.

Todo lo anterior, puede explicar el comportamiento de los cuidadores que han sufrido una LNI, porque han tenido una realidad innegable que les obliga a tener más atención al entorno.

\section{Causas volitivas reales}

La prevención no es sólo cuestión de informar o de dotar de recursos necesarios para implementarlos en el hogar. Tampoco es de mayor peso el nivel socioeconómico o la escolaridad, porque incluso cuando se pudieran regalar los recursos para brindar una mayor seguridad en el hogar, en el transporte, o en el medio donde el niño se desenvuelve, éstos pudieran no utilizarse debido a las "causas volitivas reales"; en la Tabla 3 se señalan los conceptos vertidos en los grupos focales. Por tanto, tienen un peso mayor para detonar actitudes negativas en torno a la prevención y moldear la disposición, e incluso la voluntad para actuar.

De acuerdo a esto, se sugiere que los niveles de abordaje en las estrategias de campañas futuras de prevención estén destinados a: 1) generar credibilidad en las acciones preventivas, en la certeza de que la prevención evita las LNI; y 2) en el daño que implica burlarse o ignorar los peligros o amenazas. En ambos casos, se prioriza el cuidado y seguridad del niño, independientemente de la imagen social.

Las limitaciones de este estudio pueden atribuirse a no considerar diferencias por grupo social, aunque las LNI se presentan indistintamente en cualquier nivel socioeconómico, aunque con distinta frecuencia. Otro aspecto sería la edad de los cuidadores, aunque si bien la edad pudiera atribuir conocimiento, es la experiencia de una LNI la que en este estudio demostró modificar la representación social de la prevención. Aun cuando no se dividió por géneros a los grupos focales, sí se mencionó que la mujer es quien pareciera tener mayor atención en el cuidado de los niños.

Sería importante realizar estudios donde se apliquen medidas de prevención basadas en aspectos socioculturales y de representaciones sociales para modificar la forma de ver la prevención y, por lo tanto, actuar para evitar una LNI antes de que sea necesario vivir la experiencia, a fin de tomar acciones para prevenir.

\section{CONCLUSIONES}

Las representaciones sociales de las LNI en cuidadores de niños forman parte de la cultura y definen formas de

Tabla 3: Creencias o alusiones al concepto de la prevención (causas volitivas reales).

Lo que se escucha decir en la vida cotidiana y que motiva a actuar en cierta forma (motor)

- Sobre la marcha, como vaya saliendo

- El mundo privilegia al poderoso, al valiente, al arriesgado

- Antes no usábamos sillitas y estamos vivos

- Se incentiva pensar en positivo ser optimista

- Negamos el dolor, no nos gusta el sufrimiento

- Creencia de "a mí no me pasa"

- No hay confianza en la prevención, no se cree que funcione

Lo que se hace a partir de lo que se escucha (acción)

- No hay planeación

- No se anticipa

- No se da un paso antes

- No prevengo, me arriesgo

- Innecesario, inútil, pérdida de tiempo y dinero, ¿para qué?

- No pensar en algo que me haga sentir frágil o débil

- No pensar en peligro, daños o riesgos

- Genera falsa confianza en las acciones inseguras

- No me cuido, me expongo 
interpretar la vida. La experiencia de una LNI aporta una forma distinta de observar el peligro y, por tanto, de actuar para prevenirlo. Esto es, cuando el cuidador ha experimentado una LNI modifica la forma de percibir y pensar la prevención. Sin embargo, es importante modificar la forma de percibir la prevención antes de que suceda una LNI y no esperar a que suceda. Para ello, es necesario reconocer que las conductas de prevención están influenciadas por los significados sociales y culturales de los cuidadores, de acuerdo a su forma de vida y experiencias previas.

\section{REFERENCIAS}

1. Organización Panamericana de la Salud [Internet]. Castiglione $\mathrm{S}$, Bolis M, Concha A. Lesiones no intencionales. Legislación de América Latina sobre programas preventivos y responsabilidades. Washington, DC.: OPS; 2004. p. 96 [citado 12 ene 2021]. Disponible en: https://www1.paho.org/Spanish/DD/PUB/PO_13. pdf

2. Gorrita PRR, Barrientos LG, Gorrita PY. Factores de riesgo, funcionamiento familiar y lesiones no intencionales en menores de cinco años. Revista de Ciencias Médicas La Habana. 2016; 22(1): $42-57$.

3. Gurgel A, Monteiro A. Prevención de accidentes domésticos para niños: susceptibilidad percibida por los cuidadores. Rev Pesqui Cuid Fundam. 2016; 8(4): 5126-5135.

4. Pulido S, Castro-Osorio J, Peña M, Ariza-Ramírez DP. Pautas, creencias y prácticas de crianza relacionadas con el castigo y su transmisión generacional. Revista Latinoamericana de Ciencias Sociales. Niñez y Juventud. 2013; 11(1): 245-259.

5. Moscovici S. On social representations. In: Forgas JP. Social cognition. London, England: Academic Press; 1981.

6. Jodelet D. Representación social: un área en expansión. En: Páez D. SIDA: imagen y prevención. España: Fundamentos; 1991.

7. Giménez-Montiel G, Giménez G. Identidades sociales. Cultura y representaciones sociales. 2009 4(8); 251. [citado 13 de enero de 2021]. Disponible en: $h$ ttp://www.scielo.org.mx/scielo.php?script=sci arttext\&pid=S2007-81102010000100251\&lng=es\&tlng=es.

8. Ruiz J, Gómez I, Beltrán I, Lamus D, Leal L. Representaciones sociales de normas de tránsito, agresividad, facilidad percibida en la conducción, accidentes y multas en conductores de Bogotá, D.C. Criminalidad. 2014; 56(2): 291-307.

9. Osses-Rivera SL, Macías-Gutiérrez C, Castaño-Galeano S, Gómez del Castillo D, López-Nuñez A. Representaciones sociales e infancia: una aproximación al estado del arte sobre cuidado de la salud y desarrollo integral de la infancia. Infancia Imágenes. 2014; 13(1): 70-79. doi.org/10.14483/16579089.5451.

10. Sevilla E, Ramos-Escobedo A, Franco-Ordaz A. Percepción de riesgo sobre lesiones no intencionales en niños y niñas, México. Revista Latinoamericana de Ciencias Sociales, Niñez y Juventud. 2019; 17(2): 1-22. doi: 10.11600/1692715x.17208.

11. Martínez MM, Gutiérrez-Higuera H, Alonso-Cordero ME, Hernández-Rodríguez L. Conocimientos de un grupo de madres sobre prevención de accidentes en el hogar. Revista de Ciencias Médicas La Habana. 2015; 21(2): 335-345.

12. Giddens A. Consecuencias de la modernidad. Madrid: Alianza Editorial; 1990.

13. Gorrita-Pérez RR, Taylor-Pérez N, Utria-Martinez M. Intervención sobre factores de riesgo de accidentes y accidentes en niños menores de cinco años. Medimay. 2017; 24(2): 143-159.

14. Gómez OJ, Carrillo GM, Cárdenas DC. Estrategias para el aprendizaje significativo del cuidado humano en enfermería. Archivos de Medicina. 2017; 17(2): 437-444.

15. De Siqueira JE. El principio responsabilidad de Hans Jonas. Revista Bioethikos. 2009; 3(2): 171-193.

16. González-Gómez G. El principio de la responsabilidad de Hans Jonas a la luz de la conciencia ecológica. Nómadas. Revista Crítica de Ciencias Sociales y Jurídicas. 2015; 46: 125-146.

17. Acevedo A, Vargas F. Sociología del riesgo de Niklas Luhmann. Estudio sobre las Culturas Contemporáneas. México: Universidad de Colima. 2000, 149-157.

18. Departamento de Educación de California. Seguridad y salud preventiva en los entornos de cuidado infantil, un plan de estudios para la formación de proveedores de cuidado infantil. $3^{a}$. ed. 2018.

19. Saari J. Capítulo 56 prevención de accidentes. En: Enciclopedia de Salud y Seguridad en el Trabajo. España: Editorial Instituto Nacional de Seguridad e Higiene en el Trabajo. 2012, 1-46.

20. Candreva A, Paladino C. Cuidado de la salud: el anclaje social de su construcción estudio cualitativo. Universitas Psychologica. 2005; 4(1): 55-62.

21. Rubio-Romero JC. Ingeniería de la resiliencia: conceptos básicos del nuevo paradigma en seguridad. Notas Técnicas de Prevención. 2018; 1(132): 1-10.

22. García-Vesga MC. Domínguez de la Ossa E. Desarrollo teórico de la resiliencia y su aplicación en situaciones adversas: Una revisión analítica. Revista latinoamericana de Ciencias Sociales, Niñez y Juventud. 2013; 11(1): 63-77.

Conflicto de intereses: los autores declaran que no tienen. 